\title{
Derivation of Probability Density Function of Signal-to-Interference-Plus-Noise Ratio for the MS-to-MS Interference Analysis
}

\author{
Ho-Kyung Son ${ }^{1}$ and Che-Young Kim ${ }^{2}$ \\ ${ }^{1}$ Radio Technology Research Department, Electronics and Telecommunications Research Institute, 138 Gajeongno, \\ Yuseong-gu, Daejeon 305-700, Republic of Korea \\ ${ }^{2}$ School of the Electronics Engineering, Kyungpook National University, Sankyuk-dong, Puk-gu, Daegu 702-701, Republic of Korea \\ Correspondence should be addressed to Ho-Kyung Son; hgson@etri.re.kr
}

Received 5 August 2013; Accepted 7 October 2013

Academic Editors: A. Kaloxylos, E. Stevens-Navarro, and G. Wang

Copyright @ 2013 H.-K. Son and C.-Y. Kim. This is an open access article distributed under the Creative Commons Attribution License, which permits unrestricted use, distribution, and reproduction in any medium, provided the original work is properly cited.

This paper provides an analytical derivation of the probability density function of signal-to-interference-plus-noise ratio in the scenario where mobile stations interfere with each other. This analysis considers cochannel interference and adjacent channel interference. This could also remove the need for Monte Carlo simulations when evaluating the interference effect between mobile stations. Numerical verification shows that the analytical result agrees well with a Monte Carlo simulation. Also, we applied analytical methods for evaluating the interference effect between mobile stations using adjacent frequency bands. The analytical derivation of the probability density function can be used to provide the technical criteria for sharing a frequency band.

\section{Introduction}

In wireless communication, interference between two systems occurs when these systems operate at overlapping frequencies, in the same physical environment, at the same time, with overlapping antenna patterns. In other words, these interference problems occur because several radio systems must share a limited frequency band. New technologies continue to appear, but the frequency resources are limited. Therefore, efficient use of the limited frequencies is important. To provide a more efficient use of limited frequency resources, accurate prediction of the potential interference effects between wireless communication systems is necessary.

Recently, mobile stations have populated over indoors or outdoors environments. The indoor users are mainly located inside offices, shopping malls, cafes, subways, offices, or meeting rooms. The outdoor users are typically located in squares, sidewalks, or parking spaces. Analyzing the effects of interference in these locations is necessary since these terminal stations are often in use at the same time and produce interference to each other.
So far, the various researches associated with interference analysis between terminals of Global System for Mobile Communication (GSM), Code Division Multiple Access (CDMA), Wideband Code Division Multiple Access (WCDMA), and Long Term Evolution (LTE) have been done [1-4]. Generally, the Monte Carlo method must be conducted to capture realistic system behavior and to determine the levels of interference that will be experienced within a real system when considering mobile station to mobile station interference. In [1], the researchers assume that users are uniformly located over the network area. This is not, however, the actual situation. Instead, users in the radio cell locate inside clusters. In [2], researchers derive the capacity reduction of a WCDMA downlink due to mobile-to-mobile interference, considering nonuniform user distribution. In [3], the author evaluates the impact of user equipment to user equipment interference between LTE systems operated by different operators at adjacent frequency bands, and real user distribution is also taken into account. In [4], appropriate Block Emission Mask (BEM) baseline limits are subsequently derived via Monte Carlo simulations based on interferer terminal station 


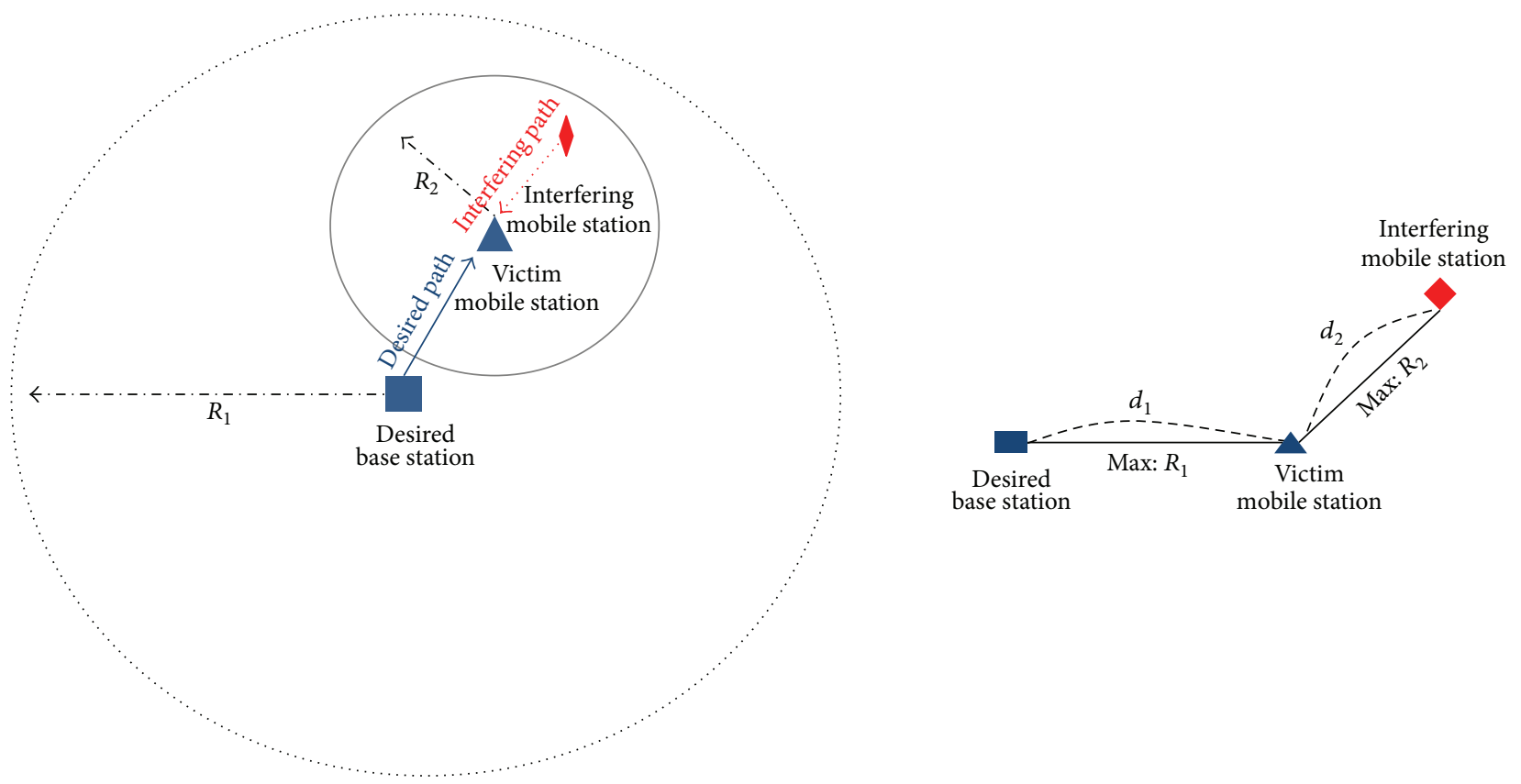

FIGURE 1: Interference model for the derivation of the pdf.

spatial densities commensurate with those observed in busy hotspots.

In this paper, we analytically derive the probability density function (pdf) of the signal-to-interference-plus-noise ratio (SINR) considering mobile-to-mobile interference in a wireless network without recourse to Monte Carlo simulations. We compare the analytical result with a Monte Carlo simulation to verify the analytical method and apply the analytical method for evaluating the interference effect of LTE's user equipment on a Trunked Radio Service (TRS)'s mobile station using adjacent frequency bands.

This paper is organized as follows. In Section 2, the interference model is described and we derive the pdf of SINR for mobile-to-mobile interference. In Section 3, the analytical method results are also compared with a Monte Carlo simulation, and the example of interference analysis using the derived analytical method is presented. Finally, the conclusions are discussed in Section 4.

\section{Analytical Derivation of the Pdf}

Figure 1 shows an interference model used to derive the pdf of SINR for analyzing mobile-to-mobile interference. We assume circular cells instead of hexagonal cells for simplicity, and the victim mobile station is at a random uniformly distributed location within the cell radius, $R_{1}$. Then we deploy interfering mobile stations at random locations within a hotspot cell radius of the victim mobile station, $R_{2}$. The pdf of SINR is calculated assuming one uniformly distributed interfering mobile station. The result obtained can be easily extended to multiple interfering mobile stations.

We also assumed that the noise power is relatively very small compared to adjacent channel interference (ACI). The ACI level is much higher than the thermal noise power level at the receiver of victim mobile station if located within the range of hotspot cell radius. The SINR in the victim mobile station can be denoted as follows:

$$
\begin{aligned}
\text { SINR } & =\frac{P_{\text {wanted }}}{P_{\text {noise }}+P_{\text {interfering }}} \\
& =\frac{C_{1}\left(d_{1}\right)^{-\alpha_{1}} \cdot 10^{P_{D} / 10}}{10^{P_{N} / 10}+10^{P_{I} / 10} \cdot 10^{-\kappa_{I} / 10} \cdot C_{2}\left(d_{2}\right)^{-\alpha_{2}}} \\
& \approx \frac{C_{1}}{C_{2}} \cdot 10^{\left(P_{D}-P_{I}+\kappa_{I}\right) / 10} \cdot \frac{d_{2}^{\alpha_{2}}}{d_{1}^{\alpha_{1}}},
\end{aligned}
$$

where $P_{D}$ is the transmitted power of the desired base station, $P_{I}$ is the transmitted power of the interfering mobile station, $P_{N}$ is the receiver thermal noise power, $\kappa_{I}$ is the adjacent channel interference ratio (ACIR) in decibels, $d_{1}$ is the distance between the victim mobile station and the desired base station to which it is connected, $d_{2}$ is the distance between the victim mobile station and the interfering mobile station, $C_{1}$ is the constant, $\alpha_{1}$ is the path loss exponent for the desired path, $C_{2}$ is the constant, and $\alpha_{2}$ is the path loss exponent for the interfering path.

Transforming the SINR in (1) into a dB scale and using the substitution $\beta=\log _{10} e$ yield

$$
\begin{aligned}
\operatorname{SINR} & =\left(10 \log _{10}\left(\frac{C_{1}}{C_{2}}\right)+P_{D}-P_{I}+\kappa_{I}\right)+10 \beta \ln \frac{d_{2}^{\alpha_{2}}}{d_{1}^{\alpha_{1}}} \\
& =K+10 \cdot \alpha_{1} \beta \ln \frac{1}{d_{1}}+10 \cdot \alpha_{2} \beta \ln d_{2},
\end{aligned}
$$

where $K$ is a constant and $d_{1}$ and $d_{2}$ are random variables. All the mobile stations are assumed to be mutually independent 
and uniformly distributed in their corresponding cells. Thus, the pdf of $d_{1}$ and $d_{2}$ are given as [5]

$$
f_{d_{i}}(x)= \begin{cases}\frac{2}{D_{i}^{2}} x, & 0 \leq x \leq R_{i} \\ 0, & \text { otherwise. }\end{cases}
$$

The pdf of random variables $y_{1}=\ln \left(1 / d_{1}\right)$ and $y_{2}=\ln \left(d_{2}\right)$ can be written as

$$
\begin{aligned}
& f_{Y_{1}}\left(y_{1}\right)= \begin{cases}f_{X_{1}}\left(x_{1}\right)\left|\frac{d x_{1}}{d y_{1}}\right|=\frac{2}{R_{1}^{2}} e^{-2 y_{1},} & -\ln \left(R_{1}\right) \leq y_{1} \leq \infty \\
0, & \text { otherwise, }\end{cases} \\
& f_{Y_{2}}\left(y_{2}\right)= \begin{cases}f_{X_{2}}\left(x_{2}\right)\left|\frac{d x_{2}}{d y_{2}}\right|=\frac{2}{R_{2}^{2}} e^{2 y_{2}}, & -\infty \leq y_{2} \leq \ln \left(R_{2}\right) \\
0, & \text { otherwise. }\end{cases}
\end{aligned}
$$

And the pdf of random variables $z_{1}=a_{1} \ln \left(1 / d_{1}\right)$ and $z_{2}=$ $a_{2} \ln \left(d_{2}\right)$ can be written as

$$
\begin{aligned}
& f_{Z_{1}}\left(z_{1}\right)= \begin{cases}\frac{2}{a_{1} R_{1}^{2}} e^{-2 z_{1} / a_{1}}, & -a_{1} \ln \left(R_{1}\right) \leq z_{1} \leq \infty \\
0, & \text { otherwise, }\end{cases} \\
& f_{Z_{2}}\left(z_{2}\right)= \begin{cases}\frac{2}{a_{2} R_{2}^{2}} e^{2 z_{2} / a_{2}}, & -\infty \leq z_{2} \leq a_{2} \ln \left(R_{2}\right) \\
0, & \text { otherwise. }\end{cases}
\end{aligned}
$$

After calculating the pdf of each random variable, the sum of the two random variables can be obtained using the following convolution [6]:

$$
\begin{aligned}
f_{Z}(z) & =\int_{-\infty}^{\infty} f_{X, Y}(x, z-x) d x \\
& =\int_{-\infty}^{\infty} f_{X}(x) f_{Y}(z-x) d x,
\end{aligned}
$$

where $f_{X}(x)$ and $f_{Y}(z-x)$ are given by

$$
\begin{aligned}
& f_{X}(x)= \begin{cases}\frac{2}{a_{1} R_{1}^{2}} e^{-2 x / a_{1}}, & -a_{1} \ln \left(R_{1}\right) \leq x \leq \infty \\
0, & \text { otherwise, }\end{cases} \\
& f_{Y}(z-x)= \begin{cases}\frac{2}{a_{2} R_{2}^{2}} e^{2(z-x) / a_{2}}, & z+a_{2} \ln \left(R_{2}\right) \leq x \\
0, & \text { otherwise. }\end{cases}
\end{aligned}
$$

If $z-a_{2} \ln \left(R_{2}\right)<-a_{1} \ln \left(R_{1}\right)$, the pdf of SINR is

$$
\begin{aligned}
f_{Z}(z) & =\int_{-a_{1} \ln \left(R_{1}\right)}^{\infty} f_{X}(x) f_{Y}(z-x) d x \\
& =\int_{-a_{1} \ln \left(R_{1}\right)}^{\infty} \frac{4}{a_{1} a_{2} R_{1}^{2} R_{2}^{2}} e^{-2 x / a_{1}+2(z-x) / a_{2}} d x \\
& =\frac{2 R_{1}^{2 a_{1} / a_{2}}}{\left(a_{1}+a_{2}\right) R_{2}^{2}} e^{\left(2 / a_{2}\right) z} .
\end{aligned}
$$

Else, if $z-a_{2} \ln \left(R_{2}\right) \geq-a_{1} \ln \left(R_{1}\right)$, the pdf of SINR is

$$
\begin{aligned}
f_{Z}(z) & =\int_{z-a_{2} \ln \left(R_{2}\right)}^{\infty} f_{X}(x) f_{Y}(z-x) d x \\
& =\int_{z-a_{2} \ln \left(R_{2}\right)}^{\infty} \frac{4}{a_{1} a_{2} R_{1}^{2} R_{2}^{2}} e^{-2 x / a_{1}+2(z-x) / a_{2}} d x \\
& =\frac{2 R_{2}^{2 a_{2} / a_{1}}}{\left(a_{1}+a_{2}\right) R_{1}^{2}} e^{-\left(2 / a_{1}\right) z} .
\end{aligned}
$$

The final pdf of SINR can be written as

$$
\begin{aligned}
f_{\text {SINR }}(t) & =f_{z}(t-K) \\
& = \begin{cases}\frac{2 R_{1}^{2 a_{1} / a_{2}}}{\left(a_{1}+a_{2}\right) R_{2}^{2}} e^{\left(2 / a_{2}\right)(t-K)}, & t<\ln \left(\frac{R_{2}^{a_{2}}}{R_{1}^{a_{1}}}\right)+K \\
\frac{2 R_{2}^{2 a_{2} / a_{1}}}{\left(a_{1}+a_{2}\right) R_{1}^{2}} e^{-\left(2 / a_{1}\right)(t-K)}, & t \geq \ln \left(\frac{R_{2}^{a_{2}}}{R_{1}^{a_{1}}}\right)+K .\end{cases}
\end{aligned}
$$

In addition, we derive the pdf of SINR by applying the shadowing model to the desired link between the desired base station and the victim mobile station. First, the expression of the SINR in (1) applied to the shadowing model is as follows:

$$
\begin{aligned}
\operatorname{SINR} & =K+\xi+10 \cdot \alpha_{1} \beta \ln \frac{1}{d_{1}}+10 \cdot \alpha_{2} \beta \ln \frac{1}{d_{2}} \\
& =X(\text { SINR without shadowing })+\xi,
\end{aligned}
$$

where $\xi$ is a random component due to shadowing on the desired path. The shadowing model is generally assumed to be log-normal distribution, so a probability distribution is the normal distribution with a standard deviation $\sigma$ and it can be expressed as follows [6]:

$$
p(\xi)=\frac{1}{\sqrt{2 \pi \sigma}} \exp \left(-\frac{\xi^{2}}{2 \sigma^{2}}\right) .
$$

Equation (11) becomes the sum of a random variable with the probability density function of (10) and random variables with normal distribution.

Then, using the convolution in (6), we can obtain the probability density function of the sum of two random variables. This can be written as follows:

$$
\begin{aligned}
f_{Z}(z)= & \frac{2 R_{2}^{2 a_{2} / a_{1}} e^{2 \sigma^{2} / a_{1}^{2}}}{\left(a_{1}+a_{2}\right) R_{1}^{2}} e^{-\left(2 / a_{1}\right)(z-K)} \\
& \times Q\left(\frac{1}{\sigma}\left(-z+\ln \left(\frac{D_{2}^{a_{2}}}{D_{1}^{a_{1}}}\right)+K+\frac{2 \sigma^{2}}{a_{1}}\right)\right) \\
& +\frac{2 R_{1}^{2 a_{1} / a_{2}} e^{2 \sigma^{2} / a_{2}^{2}}}{\left(a_{1}+a_{2}\right) R_{2}^{2}} e^{-\left(2 / a_{2}\right)(z-K)} \\
& \times Q\left(\frac{1}{\sigma}\left(-z+\ln \left(\frac{D_{2}^{a_{2}}}{D_{1}^{a_{1}}}\right)-K+\frac{2 \sigma^{2}}{a_{2}}\right)\right) .
\end{aligned}
$$

This pdf can be used to examine many different mobile-tomobile interference problems in wireless communication. 
TABLE 1: Parameters for the simulations.

\begin{tabular}{lc}
\hline Parameter & Value \\
\hline Center frequency & $900 \mathrm{MHz}$ \\
Cell radius, $R_{1}$ & $2000 \mathrm{~m}$ \\
Hotspot cell radius, $R_{2}$ & $50 \mathrm{~m}$ \\
Path loss model & Hata model \\
$\quad$ BS-MS & Motley-Keenan formula \\
$\quad$ MS-MS & $56 \mathrm{dBm}$ \\
Transmitted power of the desired base station & $24 \mathrm{dBm}$ \\
Transmitted power of the interfering mobile station & $33 \mathrm{~dB}$ \\
Adjacent channel interference ratio (ACIR) & 10,000 \\
Monte Carlo runs & \\
\hline
\end{tabular}

\section{Results and Discussion}

We compared the proposed analytical methods and a Monte Carlo simulation in order to obtain sufficient evidence as to whether both approaches lead to similar results. The Monte Carlo method has been implemented by the way of ERC report [7].

Table 1 presents the parameters used for simulation. The $900 \mathrm{MHz}$ frequency band is chosen for the simulation. The path loss model for the path between the base station and the mobile station is chosen by the Hata model [8], and the path loss model for the path between the mobile stations is selected by the Motley-Keenan formula [9]. The value $\alpha_{1}=3.52, C_{1}=$ $10^{-2}, \alpha_{2}=2$, and $C_{2}=10^{-3.15}$ can be obtained through the two path loss models. The transmitted power of the desired base station is assumed to be $56 \mathrm{dBm}$ and the transmitted power of the interfering mobile station is set to be $24 \mathrm{dBm}$. The cell radius $R_{1}$ is assumed to be $2000 \mathrm{~m}$.

Figure 2 depicts three different plots depending on the standard deviation of the log-normal shadowing model $\sigma$. The cell radius $R_{2}$ is assumed to be $50 \mathrm{~m}$ in this simulation. The results show that the analytical pdf is in agreement with the Monte Carlo simulation, and the mean of the SINR will not change as the standard deviation of the log-normal shadowing changes.

Figure 3 depicts three different plots depending on the hotspot cell radius. The standard deviation of log-normal shadowing model $\sigma$ is assumed to be $4 \mathrm{~dB}$. As the cell radius, $R_{2}$, increases, the average received interfering signal power decreases and it leads to a higher SINR mean value.

Figure 4 shows three different plots depending on the ACIR value. The standard deviation of log-normal shadowing model $\sigma$ is assumed to be $8 \mathrm{~dB}$, and the cell radius, $R_{2}$, is $50 \mathrm{~m}$. As the ACIR, $\kappa_{I}$, increases, the transmitted interfering power decreases. Therefore, the received signal power that is interfering with the other transmitter decreases, which leads to a higher SINR mean value.

The results presented in Figures 2-4 show that the analytical pdf is in agreement with the Monte Carlo simulation. Therefore, this analytical model could remove the need for Monte Carlo simulations when numerically calculating SINR.

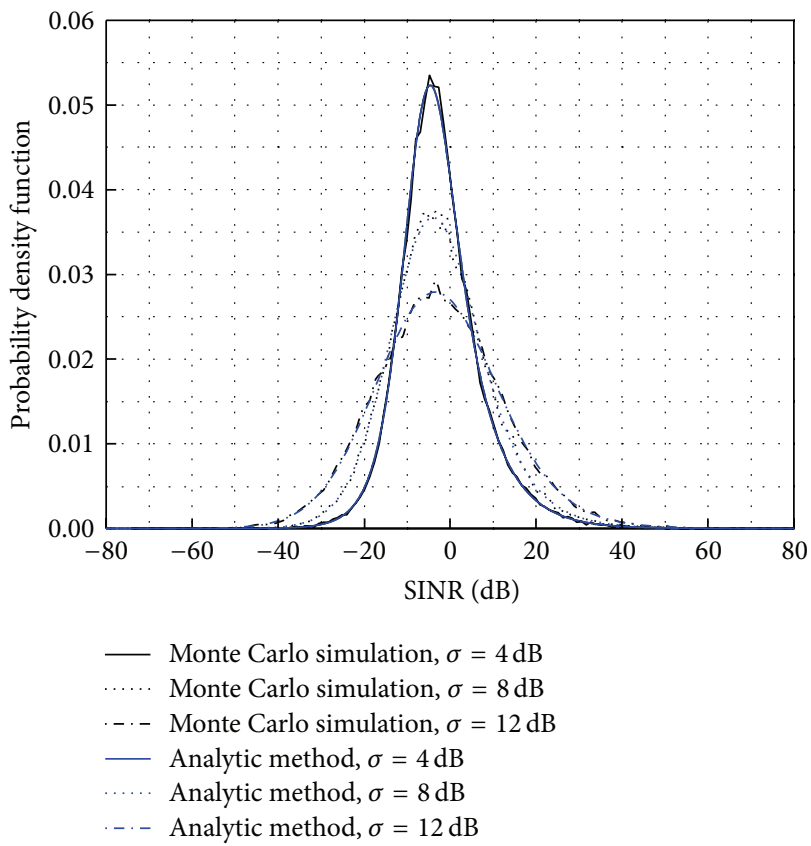

FIGURE 2: A comparison of the pdf result between the analytical method and the Monte Carlo simulation.

The following is how to apply the derived analytical method for evaluating the interference effect of LTE's user equipment (UE) on the Trunked Radio Service (TRS)'s mobile station, using an adjacent frequency band. User equipment is any device used directly by an end-user to communicate. It can be a hand-held telephone, a laptop computer equipped with a mobile broadband adapter, or any other device [10]. We assume that LTE UE is mobile station like hand-held telephone.

Figure 5 presents the cumulative density function (cdf) of SINR for different values of ACIR at the TRS's mobile station. The cdf curve can be obtained from the pdf curve using an analytical method. The parameters in Table 1 are used for the simulation and the standard deviation $\sigma$ of log-normal shadowing model is also assumed to be $8 \mathrm{~dB}$ and the hotspot cell radius $R_{2}$ is assumed to be $200 \mathrm{~m}$. The target SINR of 


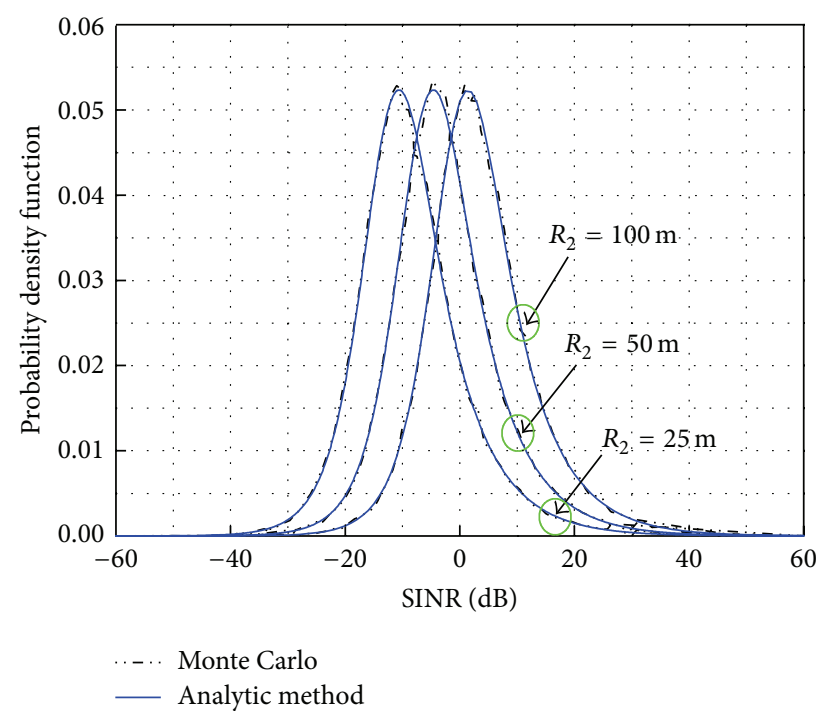

FIgURE 3: The pdf of the SINR for different values of cell radius, $R_{2}$.

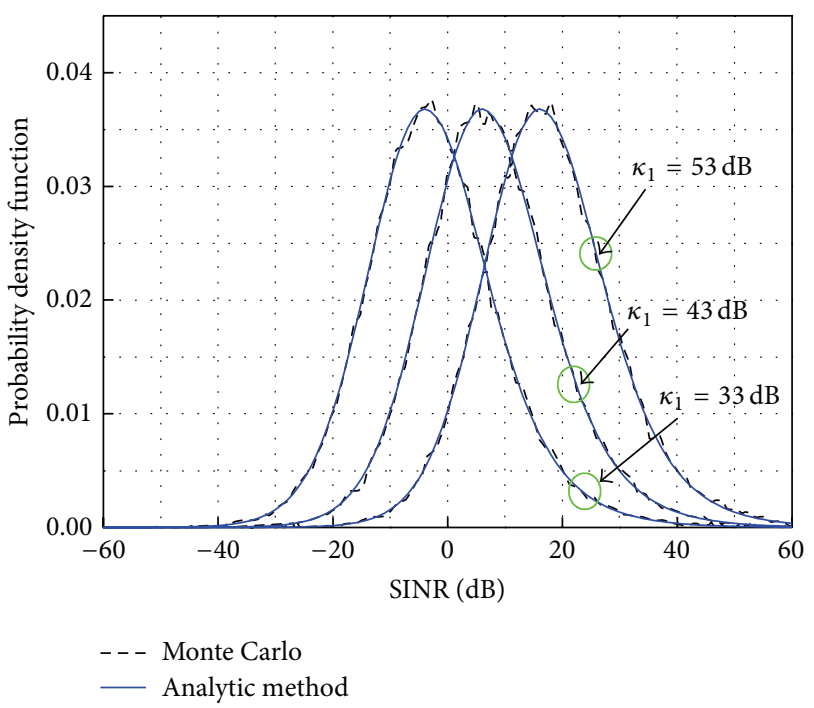

FIgURE 4: The pdf of the SINR for different values of ACIR, $\kappa_{I}$.

TRS's mobile station is assumed to be $12 \mathrm{~dB}$ [11]. The target SINR means the reference value in which TRS's mobile station may be acceptable for some degradation in audio. We say that the service outage has occurred whenever SINR of the victim receiver is dropped below to the target SINR.

At the ACIR of $\kappa_{I}=33 \mathrm{~dB}$, the probability to satisfy the target SINR is about $50 \%$ which corresponds to $50 \%$ occurrence in service outage, whereas $\kappa_{I}=53 \mathrm{~dB}$ gains a better outage probability of about $3 \%$. If the acceptable TRS's outage probability is $10 \%$, the ACIR value of about $46 \mathrm{~dB}$ can be proposed for coexistence between TRS's downlink and LTE's uplink. The requested ACIR value for coexistence increases, and it leads to a larger guard band.

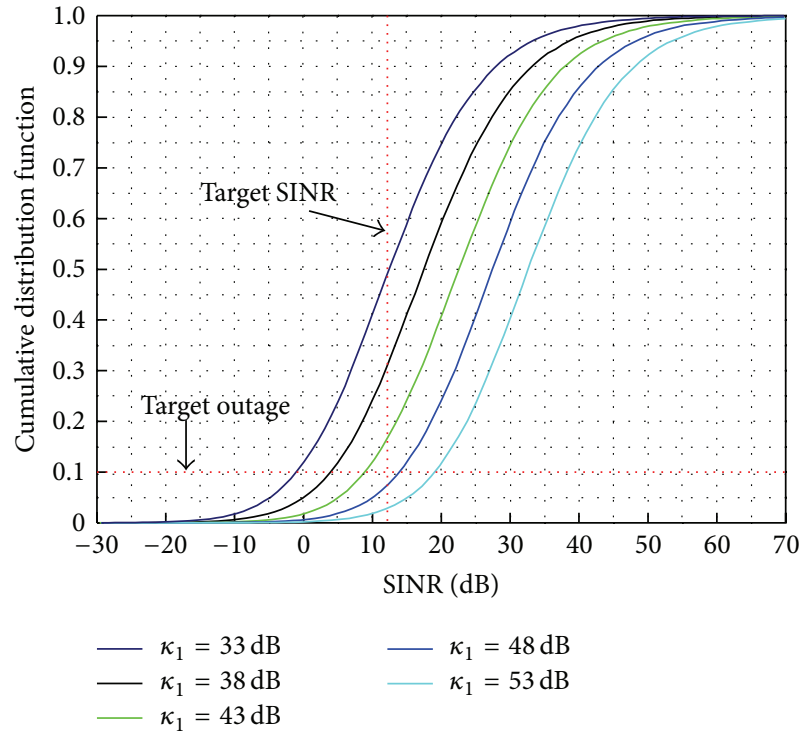

FIGURE 5: The cdf of the SINR for different values of ACIR, $\kappa_{I}$.

\section{Conclusion}

This paper described the derivation of the pdf of SINR that is used for mobile-to-mobile interference in wireless networks without Monte Carlo simulations. The analytical method is compared with a Monte Carlo simulation for the verification of the derived pdf of SINR. We confirm that the analytical pdf is in agreement with the Monte Carlo simulations. The verified analytical pdf is used to evaluate the interference effect of LTE's user equipment on the TRS's mobile station using the adjacent frequency band. We confirm that the ACIR value of about $46 \mathrm{~dB}$ can be proposed for coexistence between TRS's downlink and LTE's uplink with the acceptable TRS outage probability being $10 \%$. The proposed analytical method can be used to evaluate the interference effect between mobile stations. It can also be useful for providing technical criteria to result in an interference impact that is acceptable to both parties.

\section{Conflict of Interests}

The authors declare that there is no conflict of interests regarding the publication of this paper.

\section{Acknowledgment}

This research was funded by the Ministry of Science, ICT and Future Planning (MSIP), Republic of Korea, in the ICT R\&D Program 2013.

\section{References}

[1] H. Haas, S. McLaughlin, and G. J. R. Povey, "An investigation on capacity versus guard-bands in the TDD mode of UMTS," in Proceedings of the 52nd IEEE Vehicular Technology Conference (VTC '00), vol. 4, pp. 1820-1824, September 2000. 
[2] K. Heiska, K. Rikkinen, P. Muszynski, and U. Schwarz, "Modeling of UE-UE interference at $2.5 \mathrm{GHz}$ WCDMA," in Proceedings of the 14th IEEE International Symposium on Personal, Indoor and Mobile Radio Communications (PIMRC '03), pp. 69-73, September 2003.

[3] H. K. Son, "Throughput effects of the LTE DL due to LTE UE," International Symposium Antenna and Propagation, vol. 1, pp. 639-642, 2010.

[4] ECC, "Derivation of a block edge mask (BEM) for terminal stations in the 2. 6- GHz frequency band (2500-2690-MHz)," ECC Report 131, 2009.

[5] Y. Wang and K. Chang, "Adjacent channel interference reduction for M-WiMAX TDD and WCDMA FDD coexistence by utilizing beamforming in M-WiMAX TDD system," IEICE Transactions on Communications, vol. E93-B, no. 1, pp. 111-124, 2010.

[6] R. C. George and C. D. McGillem, Probabilistic Methods of Signal and System Analysis, Oxford University Press, New York, NY, USA, 3rd edition, 1999.

[7] ERC, "Monte-Carlo simulation methodology for the use in sharing and compatibility studies between different radio services or systems," ERC Report 68, 2002.

[8] T. S. Rappaport, Wireless Communications: Principles and Practice, Prentice Hall, Upper Saddle River, NJ, USA, 2nd edition, 2001.

[9] M. J. Nawrocki, M. Dohler, and A. H. Aghvami, Understanding UMTS Radio Network Modelling, Planning and Automated Optimisation, John Wiley \& Sons, Chichester, UK, 2006.

[10] http://en.wikipedia.org/wiki/User_equipment.

[11] R4-B26ah-0009, "Results of Monte Carlo simulations for Band 26 coexistence studies," Qualcomm Incorporated. 

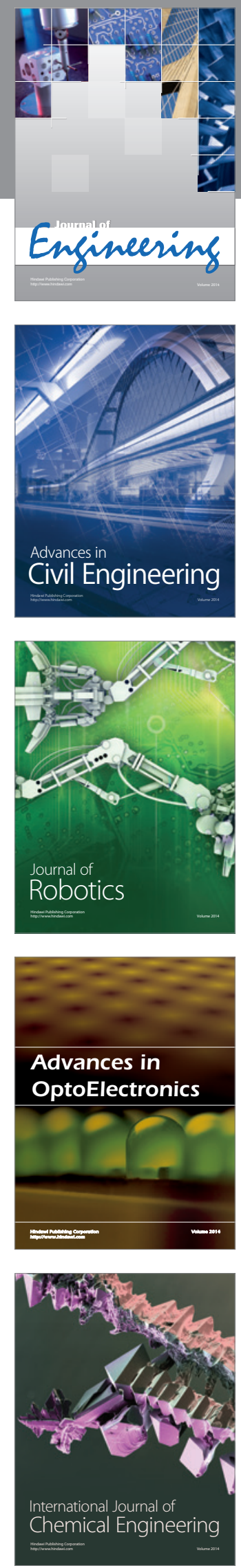

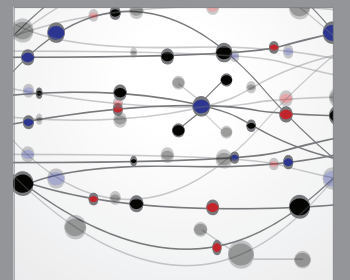

The Scientific World Journal
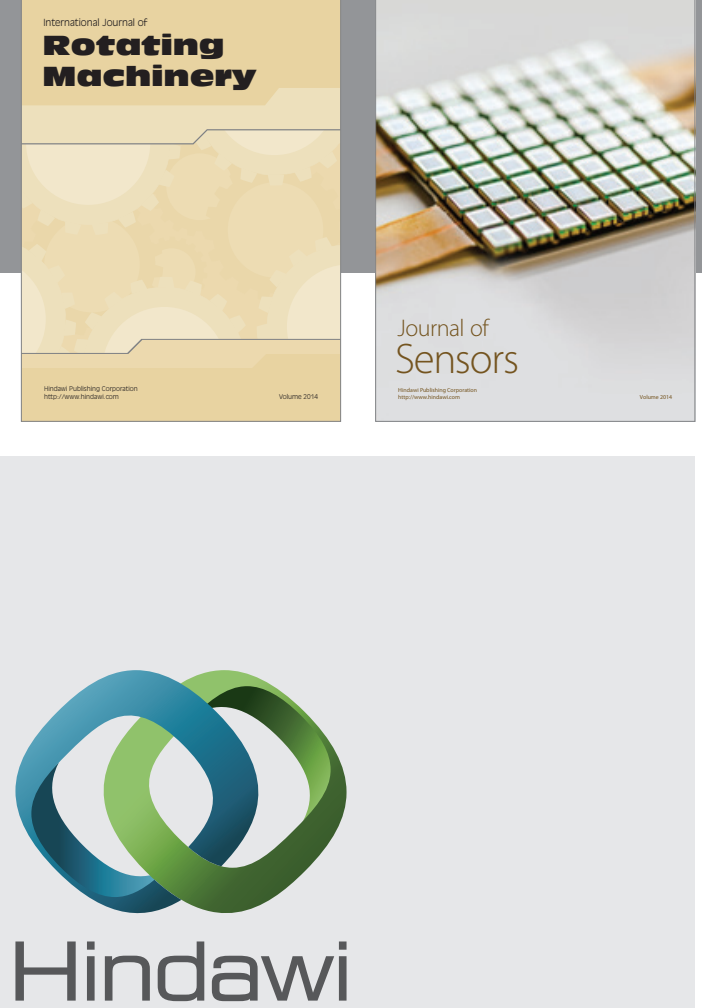

Submit your manuscripts at http://www.hindawi.com
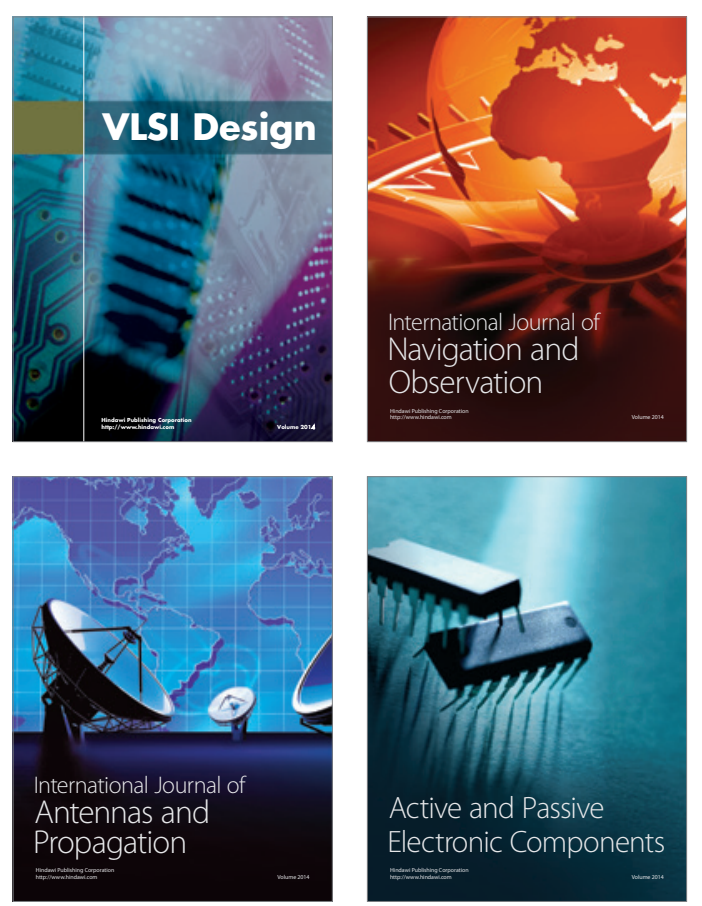
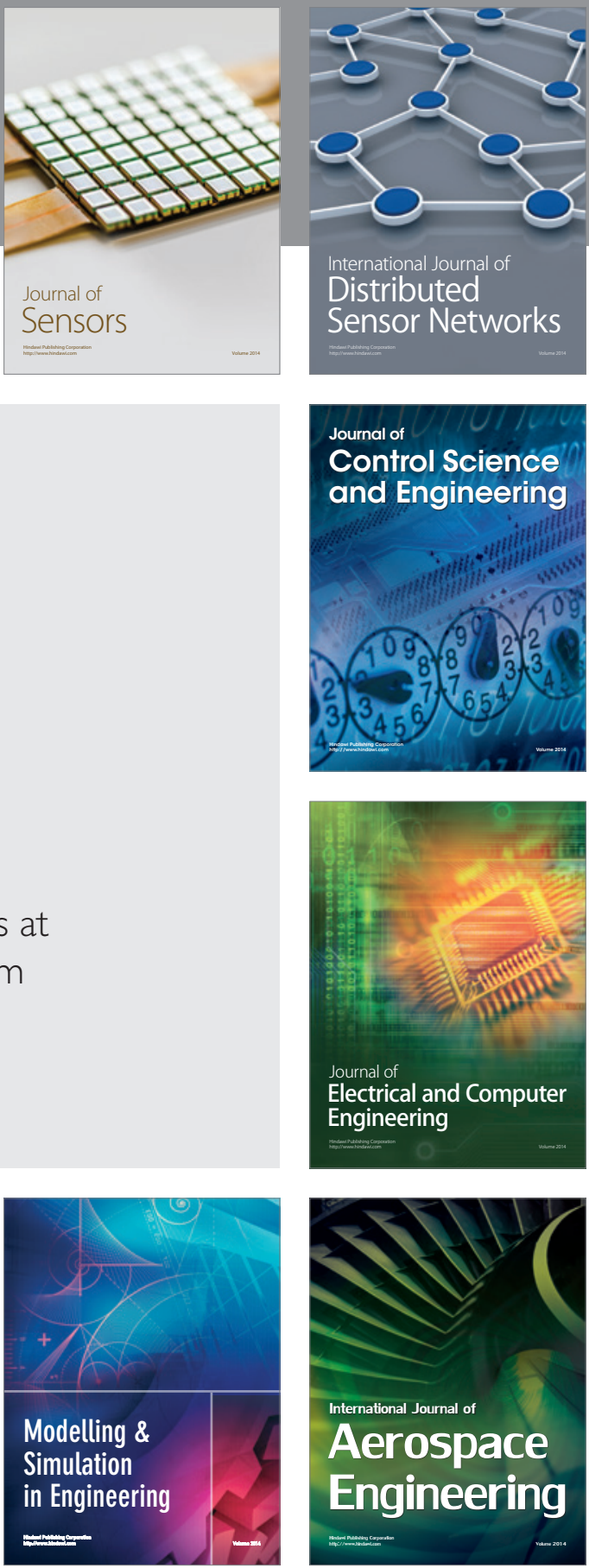

Journal of

Control Science

and Engineering
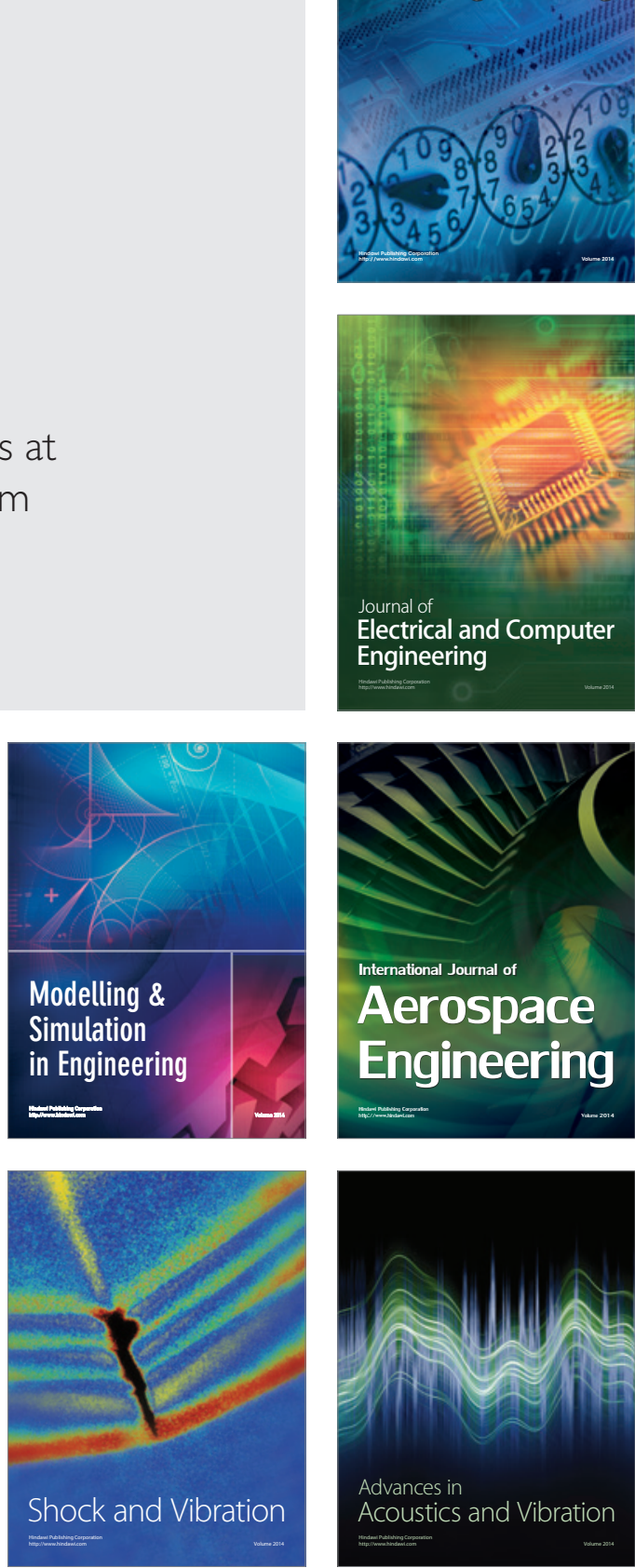